\title{
Compact hyperbolic extra dimension: a $M$-theory solution and its implications for the LHC
}

\section{Domenico Orlando and Seong Chan Park}

Institute for the Physics and Mathematics of the Universe (IPMU), The University of Tokyo, 5-1-5 Kashiwa-no-Ha, Kashiwa City, Chiba 277-8583, Japan

E-mail: domenico.orlando@ipmu.jp, seongchan.park@ipmu.jp

ABstract: We study $M$-theory solutions involving compact hyperbolic spaces. The combination of a gap à la Randall-Sundrum and the topology of an internal Riemann surface allows a geometrical solution to the hierarchy problem that does not require light Kaluza-Klein modes. We comment on the consequences of such a compactification for LHC physics.

KeYWORDS: Strings and branes phenomenology 


\section{Contents}

1 Introduction 1

$2 \quad M$-theory solutions $\quad 2$

2.1 Solutions with maximally symmetric spaces 2

2.2 Dimensional reduction 4

2.3 Stability of the breathing modes 5

3 Phenomenological implications $\quad 6$

3.14D effective action, the hierarchy problem and the Swiss Cheese Universe 6

3.2 Implications for the LHC and beyond 8

4 Discussion $\quad 10$

$\begin{array}{ll}\text { A Compact hyperbolic space } & 11\end{array}$

\section{Introduction}

An intriguing possibility is that we live in higher dimensional spacetime which contains more than three spatial dimensions. Indeed, the only candidate theories of quantum gravity are consistently constructed in $D=10$ or 11 dimensions, thus requiring six or seven extra dimensions. Even though no direct evidence of extra dimension has been found so far, several pressing problems in particle physics including the big hierarchy problem [1-3], little hierarchy problem [4], flavor hierarchy problem, neutrino mass problem, dark matter problem, supersymmetry breaking problem [5], electroweak symmetry breaking [6-8] and several others have been already addressed in the context of higher dimensions. (See e.g. [9] for a recent review).

Now the question is what the extra dimensions actually look like, how they behave and how they are stabilized. Because of lack of experimental data, theorists have considered simple model geometries like torii, spheres, (anti) de Sitter space, Calabi-Yau spaces, etc... and studied their physical consequences. In this paper we consider compact hyperbolic spaces which have been less intensively studied in physics so far [10-19] but enjoy several interesting mathematical properties (see [20,21] for a mathematical introduction). In particular, there are two properties that are of interest for our purposes:

1. A compact hyperbolic space comes equipped with two length scales: $\ell_{c}$ and $\ell_{\Gamma}$. The former, $\ell_{c}$, is related to local properties, such as the curvature and is fixed by the equations of motion (which are local in nature); the latter, $\ell_{\Gamma}$, is related to global properties, such as the volume, and is not affected by the equations of motion but appears in the expression of the effective Planck mass. 
2. The volume (and the effective Planck mass) grows exponentially with the ratio of the two scales $\ell_{\Gamma} / \ell_{c}$, thus allowing a more natural solution to the hierarchy problem.

The combination of these length scales and a Randall-Sundrum gap allows a solution to the hierarchy problem that does not require light Kaluza-Klein modes.

This paper is organized as follows. In section 2, we first report the M-theory solutions which contain a compact hyperbolic space (CHS) of the form $\mathrm{AdS}_{7-d} \times H_{d} / \Gamma \times S^{4}$ and discuss the dimensional reduction, Kaluza-Klein spectrum and the stability of the CHs. In section 3, we examine the potentially realistic solutions containing at least four noncompact dimensions, i.e. $\mathrm{AdS}_{5,4} \times H_{2,3} / \Gamma \times S^{4}$ and discuss a phenomenologically interesting large volume compactification which brings the fundamental scale of gravity down to the energies within the reach of the LHC. As one of the most interesting predictions of large volume CHS, which we call a Swiss Cheese Universe, we examine the possible production of microscopic black holes at the LHC and its future upgrade with larger energy. Differently from other cases like RS, ADD or UED models, CHS it is not necessarily accompanied by low scale Kaluza-Klein excitations of higher dimensional fields even though its volume is large. Summary and directions for future works are presented in the last section. In the appendix, we collect mathematical facts regarding CHS which are extensively used in the text.

\section{$2 \quad M$-theory solutions}

\subsection{Solutions with maximally symmetric spaces}

The bosonic part of the eleven-dimensional supergravity action is given by [22]

$$
S=\frac{M_{11}^{2}}{2} \int d^{11} x \sqrt{-G}\left(R-\frac{1}{2}|F|^{2}\right)-\frac{M_{11}^{2}}{12} \int A \wedge F \wedge F,
$$

where $A$ is the three-form gauge field and $F=\mathrm{d} A$ its associated field strength. This leads to the following equations of motion:

$$
\begin{aligned}
R_{M N} & =\frac{1}{12} F_{M N}^{2}-\frac{1}{6} G_{M N}|F|^{2}, \\
\mathrm{~d} * F+\frac{1}{2} F \wedge F & =0 .
\end{aligned}
$$

We will be interested in solutions which are direct products of Einstein spaces $\mathcal{M}_{11}=$ $\mathcal{M}^{(0)} \times \mathcal{M}^{(1)} \times \mathcal{M}^{(2)} \times \cdots$, where $\mathcal{M}^{(0)}$ contains the time coordinate. The Ricci tensor is in this case block-diagonal, with each block proportional to the corresponding metric:

$$
R_{M N}^{(a)}=k_{a} G_{M N}^{(a)} \quad \text { for } a=0,1,2, \ldots
$$

An ansatz for the four-form field strength, which solves equation (2.3) and preserves all geometric symmetries of the space, is

$$
F=\sum_{A} Q_{A} \omega^{(A)} \quad \text { with } \mathcal{M}^{(A)} \cap \mathcal{M}^{(B)} \neq \emptyset
$$


Here the $\mathcal{M}^{(A)}$ are four-dimensional sub-products inside $\mathcal{M}^{(0)} \times \mathcal{M}^{(1)} \times \mathcal{M}^{(2)} \times \cdots$, and $\omega^{(A)}$ is the corresponding volume 4-form. Note that more than one flux components are only allowed when $\mathcal{M}_{11}$ contains several factors of dimension lower than four. If $\mathcal{M}^{(A)}$ is compact, we must furthermore demand the flux-quantization condition

$$
\int_{\mathcal{M}^{(A)}} F=\frac{2 \pi n_{A}}{T_{2}}=2 \kappa^{2} T_{5} n_{A},
$$

where $T_{2}=\left(2 \pi^{2} / \kappa^{2}\right)^{1 / 3}$ is the fundamental-membrane tension, $T_{5}$ is the tension of the M-theory fivebrane, and the $n_{A}$ are integers.

The Einstein equations (2.2) reduce, with the above product ansatz, to the following set of algebraic conditions:

$$
k_{a}+R=\frac{1}{2} \sum_{A \ni a} \epsilon_{A} Q_{A}^{2} \quad \text { for } a=0,1,2, \ldots,
$$

where $R=\frac{1}{6}|F|^{2}=\sum_{a} d_{a} k_{a}$ is the total Ricci scalar, $\epsilon_{A}=+$ or - according to whether $\mathcal{M}^{(A)}$ is spacelike or timelike, and $d_{a}$ is the dimension of the factor $\mathcal{M}^{(a)}$. The sum on the right-hand side of (2.7) runs over all sub-products that contain $\mathcal{M}^{(a)}$.

Things simplify considerably when $F$ has only one non-vanishing component, in which case $\mathcal{M}_{11}=\mathcal{M}_{4} \times \mathcal{M}_{7}$, where $F=Q \times\left(\right.$ volume of $\left.\mathcal{M}_{4}\right)$. While $\mathcal{M}_{4}$ and $\mathcal{M}_{7}$ could be a priori products of simpler factors, it follows from equation (2.2) that they must themselves be Einstein manifolds (viz. $R_{i j}=k g_{i j}$ ). Slightly abusing notation, we let $k_{4}$ and $k_{7}$ be the corresponding Ricci scalars. A simple calculation gives

$$
k_{4}=\frac{Q^{2}}{3} \quad \text { and } \quad k_{7}=-\frac{Q^{2}}{6},
$$

for $\mathcal{M}_{7}$ Lorentzian, while for $\mathcal{M}_{7}$ Euclidean the signs in the above relations must be reversed. The two best-studied solutions of this type [23, 24] are the near-horizon geometries of the membrane and of the five-brane: $\mathrm{AdS}_{4} \times S^{7}$ and $\mathrm{AdS}_{7} \times S^{4}$. The fact that the equations of motion only depend on the Ricci tensor implies that $S^{4}$ and $S^{7}$ can be replaced by products of spheres, with fixed ratios of radii as imposed by the Einstein condition. Likewise, instead of $\mathrm{AdS}_{4}$ and $\mathrm{AdS}_{7}$ we may consider products of lower-dimensional AdS times hyperbolic-space factors. Explicitly, $\mathrm{AdS}_{4}$ can be replaced by $\mathrm{AdS}_{2} \times S^{2}$, and $\mathrm{AdS}_{7}$ by one of the following possibilities: $\mathrm{AdS}_{5} \times H_{2}, \mathrm{AdS}_{4} \times H_{3}, \mathrm{AdS}_{3} \times H_{4}, \mathrm{AdS}_{3} \times H_{2} \times H_{2}$, $\mathrm{AdS}_{2} \times H_{5}$ or $\mathrm{AdS}_{2} \times H_{3} \times H_{2}$. It is furthermore possible to mod out the hyperbolic factors by a group of freely-acting isometries, $H_{n} \rightarrow H_{n} / \Gamma$, so as to render them compact. It is on this type of vacua that we will focus our attention. We would like to stress once more the fact that the curvatures of the subspaces are related. In particular, for a geometry of the type $\mathrm{AdS}_{7-d} \times H_{d} \times S^{4}$, the Ricci scalars satisfy

$$
\frac{R\left[\operatorname{AdS}_{7-d 1}\right]}{7-d}=\frac{R\left[H_{d}\right]}{d}=-\frac{R\left[S^{4}\right]}{8}=-\frac{1}{\ell_{c}^{2}},
$$

where we introduced the length scale $\ell_{c}$. It is convenient to express the volumes of the compact spaces in terms of this length scale and — for the hyperbolic part — a second 
scale $\ell_{\Gamma}$ (for details see appendix A). For $d=2,3$, the volumes are

$$
V\left[S^{4}\right]=\frac{9 \pi^{2}}{8} \ell_{c}^{4}, \quad \begin{aligned}
& V\left[H_{2} / \Gamma_{g}\right] \sim \pi \ell_{c}^{2} e^{\ell_{\Gamma} / \ell_{c}}, \\
& V\left[H_{3} / \Gamma_{n}\right] \sim \pi \sqrt{2} \ell_{c}^{3} e^{\sqrt{2} \ell_{\Gamma} / \ell_{c}},
\end{aligned}
$$

where $\Gamma_{g}$ is a lattice such that $H_{2} / \Gamma_{g}$ is a genus- $g$ Riemann surface and $\Gamma_{n}=\operatorname{PSL}\left(2, \mathcal{O}_{\mathrm{n}}\right)$ as in appendix A.

These solutions are in general not protected by supersymmetry; this implies in particular that we should care about their stability. In our analyses we will deal with the breathing modes of the compact $H_{d}$ and $S^{4}$ internal manifolds which, in an effective action description, are to be represented by scalar fields. The stability (with respect to small fluctuations) will then translate into the positivity of the squared mass for such fields, a condition that can be relaxed into satisfying a Breitenlohner-Freedman bound when the space-time is of the Anti-de-Sitter type.

\subsection{Dimensional reduction}

In this section, we want to discuss the stability of the $\mathrm{AdS}_{7-d} \times H_{d} / \Gamma \times S^{4}$ solutions we found above. In particular we show that, in a consistent way, we can limit ourselves to the study of two scalar modes, corresponding to the volumes of the two compact internal manifolds. The strategy is the following: first we reduce from eleven to seven dimensions on the foursphere with flux, and then we perform a geometric reduction on the hyperbolic manifold.

To perform the first step (from eleven to seven dimensions), we need to consider the bosonic part of the seven-dimensional supergravity action [25-27]. We will use the same notation as in [25]. By construction, mass term contributions can only come from the scalars $T_{i j}$ or the 5 -plet of antisymmetric tensors $S_{\alpha \beta \gamma, I}$.

- The scalar modes are collected in the coset $\mathrm{SL}(5, \mathbb{R}) / \mathrm{SO}(5)$, hence we can choose a single representative, namely $\operatorname{det}\left(T_{i j}\right)$ which corresponds to the volume of the foursphere.

- The global SO(5) symmetry allows us to consider only one of the (spacetime) threeforms $S$. In this case, a Chern-Simons mass term could arise and be in principle negative. The relevant equation of motion reads

$$
\mathrm{d} S=\lambda * S
$$

where $\lambda$ is a symplectic Majorana spinor. Taking the Hodge dual and differentiating, one gets a Klein-Gordon equation for the topological mass term:

$$
\left(\triangle+(-)^{m r+1} \lambda^{2}\right) S=0
$$

where $\triangle$ is the Laplacian, $m=7$ is the spacetime dimension and $r=3$ is the degree of $S$. One can verify that the mass contribution is positive and does not lead to instabilities. 
The second step consists in going from seven to four dimensions. In this case, there are no gauge fields and the compactification is completely geometric. This means that KaluzaKlein instabilities can only come from scalar modes, and more precisely from the zero mode since on a compact manifold the Laplace operator is always non-negative. In other words, the only potentially dangerous mode is the overall volume of the compact manifold $H_{d} / \Gamma$. The analysis of the two modes we have identified is the object of the next section.

\subsection{Stability of the breathing modes}

Consider a metric ansatz obtained as a warped product with three factors:

$$
\mathrm{d} s^{2}=\mathrm{d} s_{M_{0}}^{2}(x)+\mathrm{e}^{2 \varphi_{1}(x)} \mathrm{d} s_{M_{1}}^{2}+\mathrm{e}^{2 \varphi_{2}(x)} \mathrm{d} s_{M_{2}}^{2},
$$

where $M_{0}, M_{1}, M_{2}$, have respectively dimension $d_{0}, d_{1}, d_{2}, M_{1}$ and $M_{2}$ are compact (we will refer to them as internal), and the $\varphi_{i}(x)$ only depend on the coordinates in $M_{0}$. We wish to study an action of the type

$$
S=\frac{M_{11}^{2}}{2} \int \mathrm{d}^{11} x \sqrt{g^{(11)}}\left(R^{(11)}-V(x)\right)
$$

where $R^{(11)}$ is the Ricci scalar in 11 dimensions and $V$ is a potential that depends on $\varphi_{i}(x)$.

Integrating out the internal coordinates one obtains an effective action in $d_{0}$ dimensions, which has to be brought to the usual Einstein-Hilbert form via a Weyl rescaling:

$$
g_{\mu \nu}^{\left(d_{0}\right)}=\exp \left[2 \frac{d_{1} \varphi_{1}+d_{2} \varphi_{2}}{d-2}\right] g_{\mu \nu}^{\left(d_{0}\right)}\left(M_{0}\right) .
$$

The contributions from the curvatures of the internal manifolds are collected into an effective potential $\bar{V}\left(\varphi_{1}, \varphi_{2}\right)$. Moreover, the scalars need to be rescaled to get the canonical dynamical term. The resulting action reads:

$$
S=\frac{M_{d_{0}}^{2}}{2} \int \mathrm{d}^{d_{0}} x \sqrt{-g^{\left(d_{0}\right)}}\left[R^{\left(d_{0}\right)}-\frac{1}{2} \partial_{\mu} \Phi_{1} \partial^{\mu} \Phi_{1}-\frac{1}{2} \partial_{\mu} \Phi_{2} \partial^{\mu} \Phi_{2}-\bar{V}\left(\Phi_{1}, \Phi_{2}\right)\right],
$$

where

$$
\bar{V}\left(\varphi_{1}, \varphi_{2}\right)=e^{-2\left(d_{1} \varphi_{1}+d_{2} \varphi_{2}\right) /\left(d_{0}-2\right)}\left(-e^{-2 \varphi_{1}(x)} R^{(1)}-e^{-2 \varphi_{2}(x)} R^{(2)}+V\left(\varphi_{1}, \varphi_{2}\right)\right),
$$

and

$$
\left\{\begin{array}{l}
\Phi_{1}=\sqrt{\frac{18}{\left(d_{1}+d_{2}\right)(d-2)}}\left(d_{1} \varphi_{1}+d_{2} \varphi_{2}\right) \\
\Phi_{2}=\sqrt{\frac{2 d_{1} d_{2}}{d_{1}+d_{2}}\left(\varphi_{1}-\varphi_{2}\right)} .
\end{array}\right.
$$

Note that $\Phi_{1}$ describes the overall volume of the compact part, and $\Phi_{2}$ describes a mode where one manifold grows while the other shrinks.

As we have stressed already, the type of backgrounds we obtain after compactification are AdS, which means that tachyons can be accepted if they don't cross the BreitenlohnerFreedman bound. It is convenient to rewrite this condition in terms of the effective potential 
$V\left(\Phi_{1}, \Phi_{2}\right)$ and its value around the stationary points. Taking into account the contribution from the negative curvature, one obtains the stability condition:

$$
-\frac{d_{0}-1}{4\left(d_{0}-2\right)}\langle\bar{V}\rangle+m_{i}^{2} \geq 0
$$

where $\langle V\rangle$ is the value of the potential at the critical points $\Phi_{i}=\bar{\Phi}_{i}$ and $m_{i}^{2}$ are the eigenvalues of the Hessian matrix

$$
H_{i j}=\left.\frac{\partial^{2} \bar{V}}{\partial \Phi_{i} \partial \Phi_{j}}\right|_{\Phi_{k}=\bar{\Phi}_{k}} .
$$

We are now in position to treat the $M$-theory backgrounds of the form $\operatorname{AdS}_{7-d} \times$ $H_{d} / \Gamma \times S^{4}$, where the hyperbolic space has been divided out by a discrete isometry group $\Gamma$ which makes it compact. The potential $V\left(\varphi_{1}, \varphi_{2}\right)$ is due to the presence of a four-form field on the $S^{4}$ part,

$$
V\left(\varphi_{1}, \varphi_{2}\right)=\frac{Q^{2}}{2} e^{-8 \varphi_{2}}
$$

The effective potential in eq. (2.18) becomes:

$$
\bar{V}\left(\varphi_{1}, \varphi_{2}\right)=e^{-2\left(d \varphi_{1}-4 \varphi_{2}\right) /(5-d)}\left(-\frac{d}{2} e^{-2 \varphi_{1}}-2 e^{\varphi_{2}}+\frac{Q^{2}}{2} e^{-8 \varphi_{2}}\right),
$$

and one finds that for any value of $d$ the solution we found above is a minimum and thus stable without having to invoke any BF arguments.

\section{Phenomenological implications}

\subsection{D effective action, the hierarchy problem and the Swiss Cheese Universe}

Among the M-theory backgrounds of the form $\mathrm{AdS}_{7-d} \times H_{d} / \Gamma \times S^{4}$, two cases are particularly interesting from the phenomenological point of view as they contain four or more non-compact dimensions:

- case i: $\mathrm{AdS}_{5} \times H_{2} / \Gamma \times S^{4}$,

- case ii: $\mathrm{AdS}_{4} \times H_{3} / \Gamma \times S^{4}$.

Of these two cases, the former is more promising as the flat $4 \mathrm{D}$ space (visible universe) can be embedded into $\mathrm{AdS}_{5}$ just like in Randall-Sundrum models [3, 28]. For the latter case to be realistic, the $\mathrm{AdS}_{4}$ space would have to be deformed to a nearly flat space by some (yet unknown) mechanism.

For a given background, we can get the 4D effective action after dimensional reduction, as discussed in the previous section. First of all, the resulting effective action contains the conventional Einstein-Hilbert term,

$$
S_{4}=\int \mathrm{d}^{4} x \sqrt{g^{(4)}} \frac{M_{4}^{2}}{2} R^{(4)}+\ldots,
$$


where the Planck scale in $4 \mathrm{D}, M_{4}=1 / \sqrt{8 \pi G_{N}}=2.435 \times 10^{18} \mathrm{GeV}$, is related to the fundamental gravity scale in eleven dimensions, $M_{11}$, as

$$
M_{4}^{2}=M_{11}^{9} \ell_{c}^{7} \mathcal{V}_{\Gamma}
$$

Here $\ell_{c}$ denotes the curvature radius of compact spaces and $\mathcal{V}_{\Gamma}$ the dimensionless volume of the CHS. As explained in appendix $\mathrm{A}, \mathcal{V}_{\Gamma}$ depends only on the topology via the scale $\ell_{\Gamma}$ :

$$
\mathcal{V}_{\Gamma}= \begin{cases}\frac{9 \pi^{3}}{8} e^{\ell_{\Gamma} / \ell_{c}} & \text { for } H_{2} / \Gamma, \\ \frac{9 \sqrt{2} \pi^{3}}{8} e^{\sqrt{2} \ell_{\Gamma} / \ell_{c}} & \text { for } H_{3} / \Gamma .\end{cases}
$$

Since $\mathcal{V}_{\Gamma}$ is not bounded from above, in principle, $M_{11}$ can be arbitrarily small. On the other hand, for both compact manifolds the first eigenvalue of the Laplacian is fixed by $\ell_{c}$ as follows:

$$
\Delta_{1}\left[H_{2} / \Gamma\right]=\frac{1}{4 \ell_{c}^{2}}, \quad \Delta_{1}\left[H_{3} / \Gamma\right]=\frac{3}{8 \ell_{c}^{2}}, \quad \Delta_{1}\left[S^{4}\right]=\frac{2}{3 \ell_{c}^{2}} .
$$

This means that $\ell_{c}^{-1}$ must be big enough to explain why the extra dimensions have not been experimentally accessible yet. More precisely, since no Kaluza-Klein (KK) excitation of the graviton has been observed, the lightest KK state, of order $\ell_{c}^{-1}$, must be heavier than $1 \mathrm{TeV}$ (the details depending on the scenario). A particularly interesting situation is realized when the gravity scale is fairly close to the electroweak scale which is the only energy scale entering in the standard model as an input. ${ }^{1}$ For instance, $M_{11} \sim 1 \mathrm{TeV}$ can be obtained provided that $\ell_{c}^{-1} \sim 1 \mathrm{TeV}$ and

$$
\mathcal{V}_{\Gamma} \sim M_{4}^{2} / \mathrm{TeV}^{2} \sim 10^{30} \Rightarrow \ell_{\Gamma}= \begin{cases}\sim 66 / \mathrm{TeV} & \text { for } H_{2} / \Gamma \\ \sim 46 / \mathrm{TeV} & \text { for } H_{3} / \Gamma\end{cases}
$$

In this case the big hierarchy between Planck scale and electroweak scale is understood as a consequence of the topology of the $\mathrm{CHS}^{2}{ }^{2}$ Since (in the two dimensional case) the genus of the surface grows exponentially with the ratio $\ell_{\Gamma} / \ell_{c}$ (as shown in appendix A), one can say that the large number of "holes" in the extra dimension is responsible for the large hierarchy. In other words, gravity is weak because we live in a "Swiss Cheese Universe".

There are two different ways of embedding flat four dimensional space in $\mathrm{AdS}_{5}$ à $l a$ Randall-Sundrum: RS-1 [3] and RS-2 [28]. In particular, in an RS-1 construction, the presence of a large warp factor provides a different explanation for the hierarchy, related to the exponential red-shift in scales. In RS-1, two branes are introduced at the boundaries $(y=0$ and $y=\ell_{5}$ ) of a slice of $\mathrm{AdS}_{5}$ described by the line element $d s^{2}=e^{-y / \ell_{c}} \eta_{\mu \nu} d x^{\mu} d x^{\nu}+d y^{2}$. Since the energy scale in the warped space depends on the location as $\Lambda(y)=e^{-y /\left(2 \ell_{c}\right)} M_{U V}$, the brane located at $y=0$ is called UV-brane and the other brane at $y=\ell_{5}$ is called

\footnotetext{
${ }^{1} \mathrm{QCD}$ scale may be regarded as another scale but it is dynamically realized by strong interactions.

${ }^{2}$ See also [12] where a similar discussion has been carried out in a completely phenomenological way. In this sense, this paper fills a theoretical gap.
} 
IR-brane. Defining $M_{U V}=M_{5}$ (the five-dimensional gravity scale), the IR scale is exponentially suppressed as $M_{I R}=M_{5} e^{-\ell_{5} /\left(2 \ell_{c}\right)}$. Here $M_{5}$ is further related to $M_{11}$ by the relation $M_{5}^{3}=M_{11}^{9} \ell_{c}^{6} \mathcal{V}_{\Gamma}$, so that

$$
M_{I R}=M_{5} e^{-\ell_{5} /\left(2 \ell_{c}\right)} \sim 3.26 M_{11}^{3} \ell_{c}^{2} \exp \left[\frac{2 \ell_{\Gamma}-3 \ell_{5}}{6 \ell_{c}}\right] .
$$

This result is interesting as "anarchy", $M_{I R} \sim M_{11} \sim \ell_{c}^{-1}$, is realized when

$$
2 \ell_{\Gamma} \sim 3 \ell_{5} .
$$

In this case, the hierarchy between $M_{5}$ and $M_{I R}$ or $M_{11}$ originates from the large warp factor and the volume of the compact hyperbolic space. Using now the fact that $M_{4}$ and $M_{5}$ are related by dimensional reduction,

$$
M_{4}^{2}=2 M_{5}^{3} \ell_{c}
$$

we can find the values of $\ell_{5}$ and $\ell_{\Gamma}$ that are required to reproduce the desired energy scales:

$$
\frac{M_{5}}{M_{11}} \sim 10^{10} \sim \mathcal{V}_{\Gamma}^{1 / 3} \sim e^{\ell_{5} /\left(2 \ell_{c}\right)} \Rightarrow \ell_{5} \sim 44 / \mathrm{TeV} .
$$

Let us stop a moment and summarize what we have seen so far. The compactification on $H_{2} / \Gamma \times S^{4}$ brings two length scales, $\ell_{c}$ and $\ell_{\Gamma}$. The former is related to the elevendimensional Planck mass $M_{11}=\ell_{c}^{-1}$. Since it appears in the expression for the KaluzaKlein modes, $\ell_{c}$ is fixed experimentally to be $\ell_{c} \geq 1 \mathrm{TeV}$. The other scale, $\ell_{\Gamma}$, governs the compactification volume and appears in the expression for the five-dimensional Planck mass. In a RS- 1 scenario, we have yet another scale, $\ell_{5}$, which contributes to the IR gravity scale in four dimensions. When the two scales are related by $2 \ell_{\Gamma} \sim 3 \ell_{5}$ an anarchy is realized, where the eleven-dimensional Planck mass $M_{11}$ and the infrared gravity scale $M_{I R}$ are both of the same order of magnitude as $\ell_{c}^{-1} \sim 1 \mathrm{TeV}$. Requiring the four-dimensional Planck mass to be of order $M_{4} \sim 2.4 \times 10^{15} \mathrm{TeV}$ fixes the values of the two length scales to $\ell_{\Gamma} \sim 66 / \mathrm{TeV}$ and $\ell_{5} \sim 44 / \mathrm{TeV}$, and gives a five-dimensional Planck mass of order $M_{5} \sim 1.4 \times 10^{10} \mathrm{TeV}$.

As a last comment we would like to point out that in a RS-2 scenario, gravity is trapped at $y=0$ and no particular relation to the IR scale is realized by the warped geometry. The hierarchy is obtained again by $\ell_{\Gamma} \sim 66 / \mathrm{TeV}$ as we have already seen.

\subsection{Implications for the LHC and beyond}

The low scale for $M_{11}$ in the Swiss Cheese Universe opens exciting possibilities for experimental tests at the LHC. It is instructive to compare our case with the well known extra dimensional scenarios, e.g., ADD [1, 2, 4], RS [3, 28] and UED [29-31] (see also [32]) since some of the features are common while others are quite distinctive.

- $M_{G} \sim 1 \mathrm{TeV}$ : Gravity becomes strong at low energy as in ADD and RS. We do not have a big hierarchy problem. In UED, $M_{5} \sim\left(M_{4}^{2} / R\right)^{1 / 3} \gg 1 \mathrm{TeV}$. 
- $M_{\mathrm{KK}} \sim 1 \mathrm{TeV}$ : Differently from ADD, our case does not allow very light KK states thus gets less constrained by cosmological and astrophysical observations. This is rather similar to the RS-1 and UED cases.

- $M_{G} \sim M_{\mathrm{KK}}$ : The effect of strong gravitation is not accompanied by KK gravitons. This feature is unique in our case since still at least a few KK gravitons or KK states of standard model fields can be seen in RS-1 or UED.

- Microscopic black holes: Once we access the transplanckian region $\sqrt{s} \gg M_{11}$, a semiclassical description of the scattering process based on eleven dimensional (super)gravity theory becomes valid [33, 34]. In fact, the Schwarzschild radius for a colliding particles with the CM energy $\sqrt{s}$ in eleven dimension,

$$
R_{\mathrm{Sch}}=\left(\frac{8}{3} \frac{\sqrt{s}}{M_{11}}\right)^{1 / 8} \frac{1}{M_{11}}
$$

is much larger than the eleven dimensional Planck length $M_{11}^{-1}$. With the expected $\mathrm{CM}$ energy $\sqrt{s}=10(14) \mathrm{TeV}$ at the LHC and the even higher energy $\sqrt{s}=100 \mathrm{TeV}$ at the future upgraded collider (VLHC), we expect that eleven dimensional microscopic black holes can be produced if $M_{11}$ is sufficiently low or $\mathcal{V}_{\Gamma}$ is big enough [35, 36]. The parton level production cross section $\hat{\sigma}$ is estimated using the Hoop conjecture, which is in good agreement with the numerical estimation [37, 38]:

$$
\hat{\sigma} \simeq F_{11}\left(\frac{\sqrt{\hat{s}}}{M_{4}}\right)^{1 / 4} \frac{\mathcal{V}_{\Gamma}^{9 / 8}}{M_{4}^{2}} \simeq \frac{1.9 \times 10^{-33}}{1 \mathrm{TeV}^{2}}\left(\frac{F_{11}}{45}\right)\left(\frac{\sqrt{\hat{s}}}{10 \mathrm{TeV}}\right)^{1 / 4} \mathcal{V}_{\Gamma}^{9 / 8},
$$

where $F_{11}$ is the form factor ${ }^{3}$ and $\sqrt{\hat{s}}$ is the CM energy in the parton frame (we assume $M_{11} \sim \ell_{c}^{-1}$ ). Even though there is a large Planck energy suppression, in principle, the large volume of hyperbolic space can overcome it and the cross section can be sizable. In figure 1 , we plot the black hole production cross section by protonproton collisions at the LHC $(\sqrt{s}=14 \mathrm{TeV}$, solid $)$ and VLHC $(\sqrt{s}=100 \mathrm{TeV}$, dotted $)$, respectively. We introduce a convenient parameter $\hat{V}$ which is defined by the relation $M_{4} / \sqrt{\mathcal{V}}=1 \mathrm{TeV} / \sqrt{\hat{V}}$ and consider the most interesting region $\sqrt{\hat{V}} \in[0.1,10]$. The cross section grows fast as $\hat{V}$ becomes bigger since the parton level cross section is proportional to $\hat{V}^{9 / 8}$ and the scale of the strong gravity so as the threshold energy is lower with larger $\hat{V}$. The minimum mass is chosen to be of the order of $10 \times M_{4} / \sqrt{\mathcal{V}}$ in this calculation. Since the KK scale is high in CHS, the dominant decay mechanism is Hawking radiation to the zero modes or the Standard Model particles. Grey-body factors for these particles have been developed in [39-44] and also Monte-Carlo event generators are available $[45,46]$.

- String winding modes: Naively one would expect that a relatively high KK scale might imply the existence of low energy winding states. However, for a Riemann surface,

\footnotetext{
${ }^{3} F_{4+n}=\pi\left(\frac{2}{\left(1+(n+2)^{2} / 4\right)^{1 /(n+1)}}\right)^{2}\left(\frac{\Gamma(n+3 / 2)}{(n+2) \pi^{(n+3) / 2}}\right)^{2 /(n+1)}$ is derived taking the angular momentum into account in $D=4+n$ dimensions.
} 


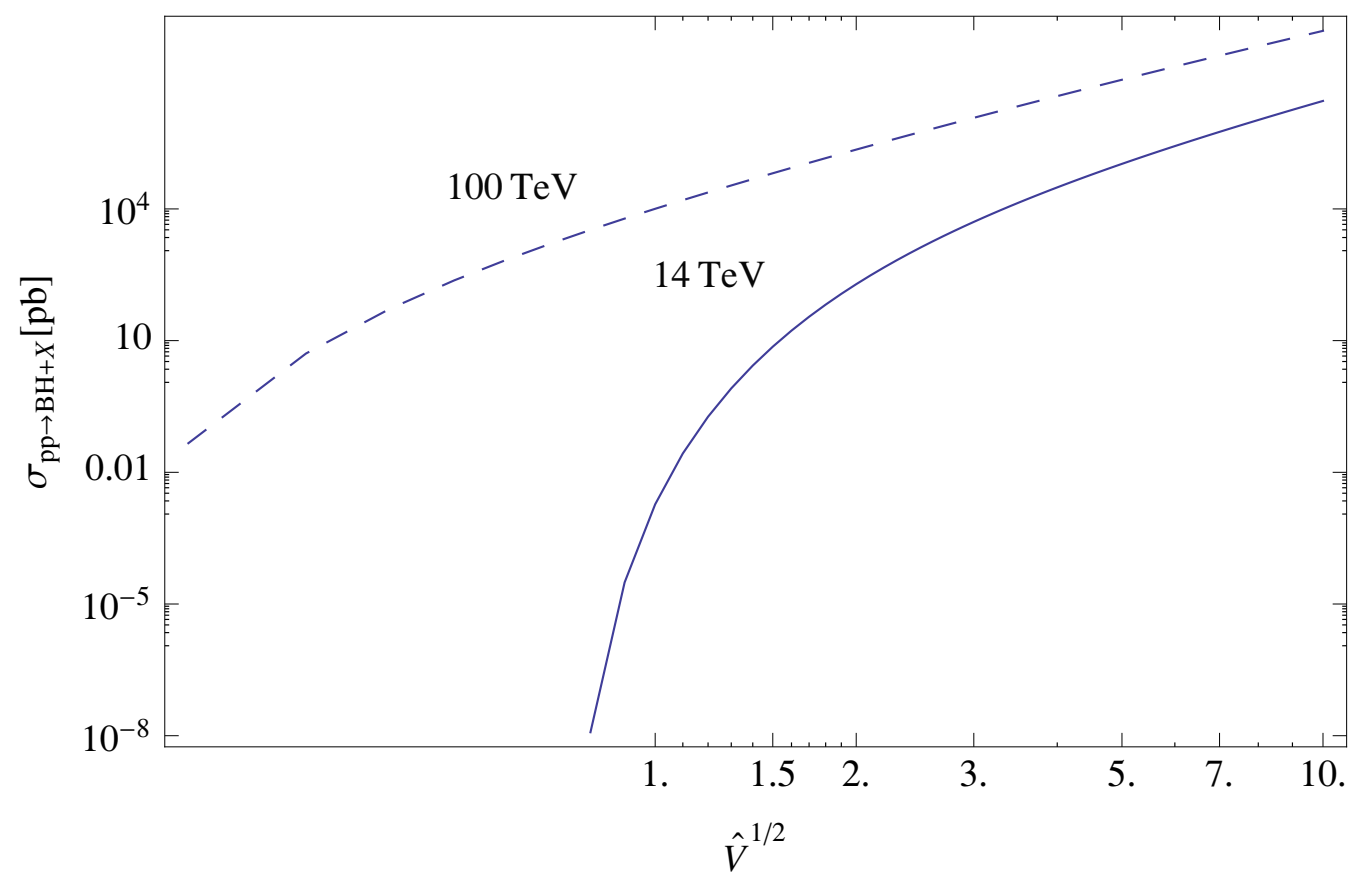

Figure 1. Production cross-sections of black hole at the LHC $(\sqrt{s}=14 \mathrm{TeV})$ and VLHC $(\sqrt{s}=$ $100 \mathrm{TeV})$.

the scale $\ell_{\Gamma}$ and the length of the shortest non-trivial geodesic $m$ are related to the volume $V$ by $[47,48]$ :

$$
m \ell_{\Gamma} \sim V .
$$

This means that the shortest path around the holes grows exponentially with $\ell_{\Gamma} / \ell_{c}$, like the volume itself. It follows that the energy of the winding modes is of order $E_{\text {wind }} \sim \tau \ell_{c}^{2} / \ell_{\Gamma} \mathcal{V}$, where $\tau=1 / l_{s}^{2}$ is the string tension, which is much bigger that the other scales we have considered so far.

\section{Discussion}

In this note, we have shown that $\mathrm{AdS}_{5} \times H^{2} / \Gamma \times S^{4}$ is a solution to eleven dimensional $M$-theory. This leads to a gap, $\ell_{5}$, in the fifth dimension of $\mathrm{AdS}_{5} \grave{a}$ la Randall-Sundrum, and a large volume for compact hyperbolic space (fixed by the topology). We can speculate that all the scales of physics, namely (i) the Planck scale $\left(M_{\text {Planck }}=\frac{1}{\sqrt{8 \pi G}}\right)$, (ii) a high scale which might be responsible for the coupling unification $\left(M_{\mathrm{GUT}}<M_{\text {Planck }}\right)$ and (iii) the scale of electroweak symmetry breaking $\left(M_{\mathrm{EW}}\right)$ are genuinely realized via the geometrical properties of higher dimensions starting from a single scale, the fundamental scale of eleven 
dimensional supergravity $M_{11} \sim 1 / \ell_{c}$ as follows:

$$
\begin{aligned}
M_{\text {Planck }} & =M_{4} \sim M_{11}^{4} \ell_{c}^{3} e^{\ell_{\Gamma} /\left(2 \ell_{c}\right)}, \\
M_{\mathrm{GUT}} & =M_{5} \sim M_{11} e^{\ell_{5} /\left(2 \ell_{c}\right)}, \\
M_{\mathrm{EW}} & =M_{11},
\end{aligned}
$$

where the topological information on the CHS is encoded in $\ell_{\Gamma}$.

Since a large $\ell_{\Gamma}$ corresponds to a large genus for CHS, we may call this spacetime the Swiss Cheese Universe.

The Swiss Cheese Universe predicts low energy strong gravity but without light KaluzaKlein excitation or light stringy winding states. Thus, the observation of microscopic black holes at high energy colliders, even possibly at the LHC, without low scale exotic KaluzaKlein particles would be a clear signature of the Swiss Cheese Universe which we believe should be examined in further detail.

\section{Acknowledgments}

We would like to thank Susanne Reffert for dicussions. DO would like to thank Costas Bachas, Costas Kounnas and Marios Petropoulos for collaboration on a related project.

This work is supported by the World Premier International Research Center Initiative (WPI initiative) by MEXT. SCP is also supported by the Grant-in-Aid for scientific research (Young Scientists (B) 21740172) from JSPS, Japan.

\section{A Compact hyperbolic space}

An $n$-dimensional maximally symmetric (Einstein) space is a pseudo-sphere in $n+1$ dimensions, i.e. the locus of the points in $\mathbb{R}^{n+1}$ satisfying the equation:

$$
\epsilon_{0}\left(X^{0}\right)^{2}+\left(X^{1}\right)^{2}+\cdots+\left(X^{n-1}\right)^{2}+\epsilon_{n}\left(X^{n}\right)^{2}=\epsilon L^{2},
$$

where the three $\epsilon$ parameters are signs. In particular, $\epsilon_{0}$ and $\epsilon_{n}$ specify the signature of the embedding $\mathbb{R}^{n+1}$ space. The hyperbolic space $H_{n}$ is the Euclidean manifold corresponding to the choice $\epsilon_{0}=-1, \epsilon_{n}=1, \epsilon=-1$ (other choices of the signs lead to $\mathrm{AdS}_{n}, \mathrm{dS}_{n}$ or $S^{n}$ ).

By construction $H_{n}$ has (negative) constant curvature. In terms of Riemannian geometry, the relevant tensors can be written as:

$$
\begin{aligned}
R_{a b c d} & =-\frac{1}{L^{2}}\left(g_{a d} g_{b c}-g_{a c} g_{b d}\right), \\
R i c_{a b} & =R^{c}{ }_{a c b}=-\frac{1}{L^{2}}(n-1) g_{a b}, \\
R & =\operatorname{Ric}^{a}{ }_{a}=-\frac{1}{L^{2}} n(n-1) .
\end{aligned}
$$

For later convenience we also introduce a length scale $\ell_{c}$ via

$$
\ell_{c}^{2}=\frac{L^{2}}{n-1}=\frac{n}{|R|} .
$$


Another very useful description can be given in terms of Lie groups. A maximally symmetric space is identified with the coset $G / T$ (we quotient with respect to the action of $T$ on the left, $g \sim g t$ ), where $T$ is the maximal subgroup in the group $G$. In particular, one can show that

$$
H_{n}=\frac{\mathrm{SO}(1, \mathrm{n})}{\mathrm{SO}(\mathrm{n})} .
$$

The first obvious consequence is that $\mathrm{SO}(1, \mathrm{n})$ is the group of isometries of $H_{n}$.

A particularly convenient choice of coordinates, covering the whole manifold, is given by the so-called Poincaré coordinates. The line element takes the form

$$
\mathrm{d} s^{2}=g_{a b} \mathrm{~d} u^{a} \mathrm{~d} u^{b}=\frac{L^{2}}{\left(u^{1}\right)^{2}}\left(\left(\mathrm{~d} u^{1}\right)^{2}+\cdots+\left(\mathrm{d} u^{n}\right)^{2}\right)
$$

where $u^{i} \in(0,+\infty)$. In such coordinates it is evident that such manifolds have infinite volume, i.e. the integral $\left(\int \mathrm{d} u^{1} \ldots \mathrm{d} u^{n} \sqrt{g}\right)$ diverges.

Starting from $H_{n}$, it is possible to construct compact manifolds of constant negative curvature by taking the quotient with respect to the action of a freely acting discrete group $\Gamma \subset \mathrm{SO}(1, \mathrm{n})$ (from now on we call this a lattice). It is worth emphasizing that, although $H_{n}$ and $H_{n} / \Gamma$ share the same local properties, the global properties are completely different. The construction is general, in the sense that any closed manifold of negative constant curvature can be written as a quotient $H_{n} / \Gamma$.

One of the most important results in the study of these manifolds is Mostow's rigidity theorem [49]. It states that the geometry of a finite volume hyperbolic manifold of dimension greater than two is determined by its fundamental group. ${ }^{4}$ In particular, this means that once we have fixed the curvature $(L)$ and the volume, there are no more moduli. The theorem is not valid in $d=2$ dimensions. In fact, a Riemann surface of genus $g>1$ (which can always be represented as quotients $\left.H_{2} / \Gamma, \Gamma \subset \mathrm{SO}(1,2)\right)$, has a $6(g-1)$-dimensional moduli space.

In view of the following physical applications, we will now concentrate on the lowerdimensional $d=2$ and $d=3$ examples.

$\boldsymbol{d}=\mathbf{2}$, or, Riemann surfaces. Depending on their genus, Riemann surfaces can be endowed with a metric that can be spherical (genus $g=0$ ), flat (genus $g=1$ ) or hyperbolic $(g \geq 2)$.

Any surface of genus $g$ can be described in terms of a polygon. In particular we define the metric fundamental polygon as the $(4 g+2)$-gon in which the edges are pairwise identified and the standard fundamental polygon as the $(4 g)$-gon in which the edges are pairwise identified and all the vertices are identified. In the usual notation, an $n$-gon is represented as a string of $n$ letters with exponent \pm 1 depending on the orientation of the edge with respect to an arbitrary positive one. The same letter is used for pairs of identified edges. The most familiar example is the torus that can be built out of a parallelogram lattice in $\mathbb{R}^{2}$, opposite sides being identified and having opposite orientation, that is to say the standard fundamental polygon is $A B A^{-1} B^{-1}$. Equivalently, the torus is also to be obtained

\footnotetext{
${ }^{4}$ We are not considering the case of cusped hyperbolic three-manifolds or incomplete metrics.
} 
by an hexagonal lattice (metric fundamental polygon) with sides $A B C A^{-1} B^{-1} C^{-1}$.

The fundamental polygons can be seen as elementary cells for a tessellation of the hyperbolic plane $\mathrm{H}_{2}$. In figure 2 we show one such tessellation, corresponding to a genus two surface, on the Poincaré disc.

There are two properties of Riemann surfaces that will be of interest in the following: the volume, which is function of the genus $g$ and the curvature

$$
V\left[H_{2} / \Gamma_{g}\right]=4 \pi L^{2}(g-1)=8 \pi \frac{1}{|R|}(g-1),
$$

and the fact that the scalar Laplacian is massive and the gap only depends on the curvature. One can always choose a Riemann surface such that:

$$
\Delta_{1} \geq \frac{1}{4 L^{2}}=\frac{|R|}{8} .
$$

Note that there are two independent parameters that fix these two quantities: a "local" parameter $L$ and a "global" parameter $g$. This is an important difference with respect to the case of the sphere, where both volume and mass gap are fixed by the curvature. This is the key property of compact hyperbolic manifolds that allows us to propose a different mechanism for dealing with the hierarchy problem.

It is convenient to introduce a scale parameter $\ell_{\Gamma}$ related to the global properties of the surface, to express the volume. Recall that the volume of a $d$-ball of radius $\ell_{\Gamma}$ in $H_{d}$ is given by

$$
V_{d}\left(\ell_{\Gamma}\right)=S_{d-1} L^{d} I_{d-1}\left(\ell_{\Gamma} / L\right),
$$

where $S_{d-1}$ is the surface area of a Euclidean $(d-1)$-sphere and $I_{d-1}\left(\ell_{\Gamma} / L\right)$ is the integral

$$
I_{d-1}\left(\ell_{\Gamma} / L\right)=\int_{0}^{\ell_{\Gamma} / L} \sinh (\xi)^{d-1} \mathrm{~d} \xi
$$

We can then define the typical length $\ell_{\Gamma}$ of a Riemann surface of genus $g$ via:

$$
4 \pi L^{2}(g-1) \sim 2 \pi L^{2} \cosh \left(\ell_{\Gamma} / L\right) \Rightarrow l \sim L \log (g),
$$

and the volume is

$$
V\left[H_{2} / \Gamma\right] \sim \pi L^{2} e^{\ell_{\Gamma} / L}
$$

Hyperbolic three-manifolds. The three-dimensional case is the first example in which Mostow's theorem holds. In particular, this means that the geometry of a compact hyperbolic three-manifold is fixed by the choice of the lattice and hence by its volume. Here we show how this volume can be computed for a large family of lattices by using algebraic techniques.

The hyperbolic space $H_{3}$ is the coset $\mathrm{SO}(1,3) / \mathrm{SO}(3)$. This implies that its group of isometries is $\mathrm{SL}(2, \mathbb{C}) \sim \mathrm{SO}(1,3)$ and in particular any discrete lattice used to compactify is $\Gamma \subset \operatorname{SL}(2, \mathbb{C})$. A convenient choice of $\Gamma$, depending on an integer number $d$ leads to the so-called Bianchi manifolds for which it is possible to give explicit expressions for the volume and mass gap. Let $n$ be a square-free integer (i.e. $n$ does not contain any perfect 


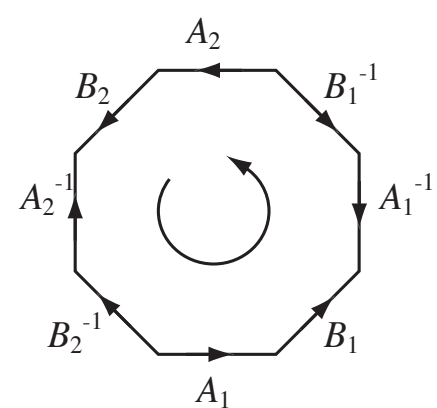

(a)

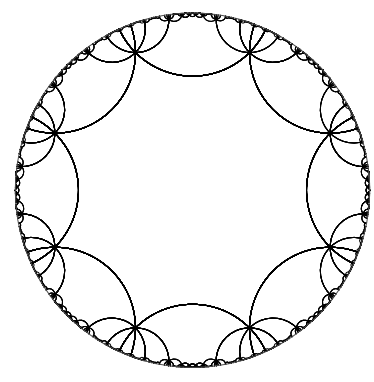

(b)

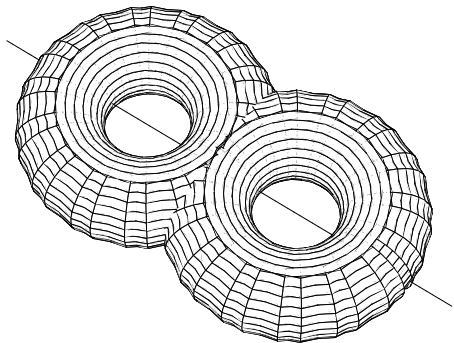

(c)

Figure 2. Different representations of a Riemann surface of genus $g=2$. (a) Standard fundamental polygon $A_{1} B_{1} A_{1}^{-1} B_{1}^{-1} A_{2} B_{2} A_{2}^{-1} B_{2}^{-1}$; (b) tessellation of the Poincaré disc; (c) embedding in $\mathbb{R}^{3}$.

square as factor). Consider the quadratic field $\mathbb{Q}(\sqrt{-n})=\{a+b \sqrt{-n} \mid a, b \in \mathbb{Q}\}$, i.e. the two-dimensional vector space on $\mathbb{Q}$ generated by 1 and $\sqrt{-n}$. Let $\mathcal{O}_{n} \subset Q(\sqrt{-n})$ be the ring of integers in this field. Explicitly:

$$
\mathcal{O}_{n}=\mathbb{Z}[\omega], \quad \omega= \begin{cases}\frac{1}{2}(-1+\sqrt{-n}) & \text { if } n=3 \bmod 4 \\ \sqrt{-n} & \text { otherwise }\end{cases}
$$

(Note that when $n=3 \bmod 4, \omega$ is an algebraic integer since it satisfies the equation $\left.\omega^{2}+\omega+\frac{(n+1)}{4}=0\right)$.

The most simple example is $\mathbb{Q}(\imath)$, the so-called the Gaussian rationals, i.e. the field $\mathbb{Q}(\imath)=$ $\{a+\imath b \mid a, b \in \mathbb{Q}\}$ and $\mathcal{O}_{1}$ is the ring of Gaussian integers $\mathcal{O}_{1}=\mathbb{Z}[\imath]=\{a+\imath b \mid a, b \in \mathbb{Z}\}$.

$\mathcal{O}_{d}$ is a lattice in $\mathbb{C}$, so we can consider the lattice $\operatorname{PSL}\left(2, \mathcal{O}_{\mathrm{n}}\right) \subset \operatorname{PSL}(2, \mathbb{C})$ and the compactifications $H_{3} / \Gamma$ where $\Gamma \subset \operatorname{PSL}(2, \mathbb{C})$. When one sees $\mathcal{O}_{n}$ as a lattice in $\mathbb{C}$, its area is given by $\operatorname{vol}\left(\mathcal{O}_{n}\right)=\sqrt{D} / 2$ where, $D$ is the discriminant:

$$
D\left(\mathcal{O}_{n}\right)= \begin{cases}n & \text { if } n=3 \bmod 4 \\ 4 n & \text { otherwise }\end{cases}
$$

We need to take into account the difference between considering the lattice on $\mathbb{C}$ and on $\operatorname{PSL}(2, \mathbb{C})$, and this is done by using the formula [50]

$$
\operatorname{vol}\left(H_{3} / \operatorname{PSL}\left(2, \mathcal{O}_{\mathrm{n}}\right)\right)=\frac{\mathrm{L}\left(\chi_{\mathrm{n}}, 2\right)}{24} \mathrm{~L}^{3} \mathrm{D}^{3 / 2}=\frac{\mathrm{L}\left(\chi_{\mathrm{n}}, 2\right)}{3}\left(\frac{3 \mathrm{D}}{2|\mathrm{R}|}\right)^{3 / 2},
$$

where $L\left(\chi_{n}, s\right)$ is the Dirichlet $L$-series for the principal character $\chi_{n}$ (for our purposes it is worth to remark that $\left.1 \leq L\left(\chi_{n}, 2\right) \leq \zeta(2)=\pi^{2} / 6 \sim 1.644\right)$. Also in this case, it is convenient to introduce a length scale $\ell_{\Gamma}$, related to the volume via

$$
\frac{L\left(\chi_{n}, 2\right)}{24} L^{3} D^{3 / 2} \sim 4 \pi L^{3}\left(\frac{\sinh \left(2 \ell_{\Gamma} / L\right)}{4}-\frac{\ell_{\Gamma}}{2 L}\right) \Rightarrow l \sim \frac{3}{4} L \log (n) .
$$


The volume of the three-manifold is given by

$$
V\left[H_{3} / \Gamma\right] \sim \frac{\pi L^{3}}{2} e^{2 \ell_{\Gamma} / L}
$$

Using number-theoretical techniques, it is possible to evaluate the mass gap. Just like in the Riemann surface case, this only depends on the global parameter $L$ and is

$$
\Delta_{1} \geq \frac{3}{4 L^{2}}=\frac{9|R|}{2} .
$$

Note that, just like before, volume and mass gap depend on two parameters $L$ and $n$ that can be adjusted independently.

\section{References}

[1] N. Arkani-Hamed, S. Dimopoulos and G.R. Dvali, The hierarchy problem and new dimensions at a millimeter, Phys. Lett. B 429 (1998) 263 [hep-ph/9803315] [SPIRES].

[2] I. Antoniadis, N. Arkani-Hamed, S. Dimopoulos and G.R. Dvali, New dimensions at a millimeter to aFermi and superstrings at a TeV, Phys. Lett. B 436 (1998) 257 [hep-ph/9804398] [SPIRES].

[3] L. Randall and R. Sundrum, A large mass hierarchy from a small extra dimension, Phys. Rev. Lett. 83 (1999) 3370 [hep-ph/9905221] [SPIRES].

[4] N. Arkani-Hamed, A.G. Cohen, E. Katz and A.E. Nelson, The littlest Higgs, JHEP 07 (2002) 034 [hep-ph/0206021] [SPIRES].

[5] L. Randall and R. Sundrum, Out of this world supersymmetry breaking, Nucl. Phys. B 557 (1999) 79 [hep-th/9810155] [SPIRES].

[6] N. Arkani-Hamed, A.G. Cohen and H. Georgi, Electroweak symmetry breaking from dimensional deconstruction, Phys. Lett. B 513 (2001) 232 [hep-ph/0105239] [SPIRES].

[7] C. Csáki, C. Grojean and H. Murayama, Standard Model Higgs from Higher Dimensional Gauge Fields, Phys. Rev. D 67 (2003) 085012 [hep-ph/0210133] [SPIRES].

[8] C. Csáki, C. Grojean, L. Pilo and J. Terning, Towards a realistic model of Higgsless electroweak symmetry breaking, Phys. Rev. Lett. 92 (2004) 101802 [hep-ph/0308038] [SPIRES].

[9] C. Csáki, TASI lectures on extra dimensions and branes, hep-ph/0404096 [SPIRES].

[10] A. Kehagias and J.G. Russo, Hyperbolic spaces in string and M-theory, JHEP 07 (2000) 027 [hep-th/0003281] [SPIRES].

[11] J.P. Gauntlett, N. Kim and D. Waldram, M-fivebranes wrapped on supersymmetric cycles, Phys. Rev. D 63 (2001) 126001 [hep-th/0012195] [SPIRES].

[12] N. Kaloper, J. March-Russell, G.D. Starkman and M. Trodden, Compact hyperbolic extra dimensions: Branes, Kaluza-Klein modes and cosmology, Phys. Rev. Lett. 85 (2000) 928 [hep-ph/0002001] [SPIRES].

[13] C.-M. Chen, P.-M. Ho, I.P. Neupane, N. Ohta and J.E. Wang, Hyperbolic space cosmologies, JHEP 10 (2003) 058 [hep-th/0306291] [SPIRES]. 
[14] I.P. Neupane, Accelerating cosmologies from exponential potentials, Class. Quant. Grav. 21 (2004) 4383 [hep-th/0311071] [SPIRES].

[15] D. Orlando, M-theory compactifications on hyperbolic spaces, Fortsch. Phys. 55 (2007) 793 [hep-th/0702013] [SPIRES].

[16] D. Orlando, String theory: Exact solutions, marginal deformations and hyperbolic spaces, Fortsch. Phys. 55 (2007) 161 [hep-th/0610284] [SPIRES].

[17] E. Silverstein, Simple de Sitter Solutions, Phys. Rev. D 77 (2008) 106006 [arXiv: 0712.1196] [SPIRES].

[18] M.R. Douglas and R. Kallosh, Compactification on negatively curved manifolds, JHEP 06 (2010) 004 [arXiv: 1001.4008] [SPIRES].

[19] B. Greene, D. Kabat, J. Levin and D. Thurston, A bulk inflaton from large volume extra dimensions, arXiv: 1001.1423 [SPIRES].

[20] H. M. Farkas and I. Kra, Riemann surfaces, Springer-Verlag, New York, U.S.A. (1980).

[21] W. Thurston, The Geometry and Topology of Three Manifolds, Princeton University Lecture Notes, Princeton U.S.A. (1978).

[22] E. Cremmer, B. Julia and J. Scherk, Supergravity theory in 11 dimensions, Phys. Lett. B 76 (1978) 409 [SPIRES].

[23] P.G.O. Freund and M.A. Rubin, Dynamics of Dimensional Reduction, Phys. Lett. B 97 (1980) 233 [SPIRES].

[24] M. J. Duff and C. N. Pope, Kaluza-Klein Supergravity and the Seven Sphere, in Super-symmetry and Supergravity 82, Ferrara, Taylor and van Nieuwenhuizen eds., World Scientific (1983).

[25] M. Pernici, K. Pilch and P. van Nieuwenhuizen, Gauged maximally extended supergravity in seven- dimensions, Phys. Lett. B 143 (1984) 103 [SPIRES].

[26] M.J. Duff, P.K. Townsend and P. van Nieuwenhuizen, Spontaneous compactification of supergravity on the three - sphere, Phys. Lett. B 122 (1983) 232 [SPIRES].

[27] P.K. Townsend and P. van Nieuwenhuizen, Gauged seven-dimensional supergravity, Phys. Lett. B 125 (1983) 41 [SPIRES].

[28] L. Randall and R. Sundrum, An alternative to compactification, Phys. Rev. Lett. 83 (1999) 4690 [hep-th/9906064] [SPIRES].

[29] I. Antoniadis, A Possible new dimension at a few TeV, Phys. Lett. B 246 (1990) 377 [SPIRES].

[30] T. Appelquist, H.-C. Cheng and B.A. Dobrescu, Bounds on universal extra dimensions, Phys. Rev. D 64 (2001) 035002 [hep-ph/0012100] [SPIRES].

[31] H.-C. Cheng, K.T. Matchev and M. Schmaltz, Radiative corrections to Kaluza-Klein masses, Phys. Rev. D 66 (2002) 036005 [hep-ph/0204342] [SPIRES].

[32] S.C. Park and J. Shu, Split-UED and Dark Matter, Phys. Rev. D 79 (2009) 091702 [arXiv: 0901.0720] [SPIRES].

[33] G. 't Hooft, Graviton Dominance in Ultrahigh-Energy Scattering, Phys. Lett. B 198 (1987) 61 [SPIRES]. 
[34] T. Banks and W. Fischler, A model for high energy scattering in quantum gravity, hep-th/9906038 [SPIRES].

[35] S.B. Giddings and S.D. Thomas, High energy colliders as black hole factories: The end of short distance physics, Phys. Rev. D 65 (2002) 056010 [hep-ph/0106219] [SPIRES].

[36] S. Dimopoulos and G.L. Landsberg, Black Holes at the LHC, Phys. Rev. Lett. 87 (2001) 161602 [hep-ph/0106295] [SPIRES].

[37] D.M. Eardley and S.B. Giddings, Classical black hole production in high-energy collisions, Phys. Rev. D 66 (2002) 044011 [gr-qc/0201034] [SPIRES].

[38] H. Yoshino and Y. Nambu, Black hole formation in the grazing collision of high-energy particles, Phys. Rev. D 67 (2003) 024009 [gr-qc/0209003] [SPIRES].

[39] D. Ida, K.-y. Oda and S.C. Park, Rotating black holes at future colliders: Greybody factors for brane fields, Phys. Rev. D 67 (2003) 064025 [hep-th/0212108] [SPIRES].

[40] D. Ida, K.-y. Oda and S.C. Park, Rotating black holes at future colliders. II: Anisotropic scalar field emission, Phys. Rev. D 71 (2005) 124039 [hep-th/0503052] [SPIRES].

[41] D. Ida, K.-y. Oda and S.C. Park, Rotating black holes at future colliders. III: Determination of black hole evolution, Phys. Rev. D 73 (2006) 124022 [hep-th/0602188] [SPIRES].

[42] C.M. Harris and P. Kanti, Hawking radiation from a $(4+n)$-dimensional rotating black hole, Phys. Lett. B 633 (2006) 106 [hep-th/0503010] [SPIRES].

[43] M. Casals, P. Kanti and E. Winstanley, Brane decay of a $(4+n)$-dimensional rotating black hole. II: Spin-1 particles, JHEP 02 (2006) 051 [hep-th/0511163] [SPIRES].

[44] M. Casals, S.R. Dolan, P. Kanti and E. Winstanley, Brane decay of a $(4+n)$-dimensional rotating black hole. III: Spin-1/2 particles, JHEP 03 (2007) 019 [hep-th/0608193] [SPIRES].

[45] D.-C. Dai et al., BlackMax: A black-hole event generator with rotation, recoil, split branes and brane tension, Phys. Rev. D 77 (2008) 076007 [arXiv:0711.3012] [SPIRES].

[46] J.A. Frost et al., Phenomenology of Production and Decay of Spinning Extra- Dimensional Black Holes at Hadron Colliders, JHEP 10 (2009) 014 [arXiv:0904.0979] [SPIRES].

[47] D. Mumford, A remark on mahler's compactness theorem, P. Am. Math. Soc. 28 (1971) 289.

[48] F.-R. Chang, On the diameters of compact riemann surfaces, P. Am. Math. Soc. 65 (1977) 274.

[49] G. Mostow, Quasi-conformal mappings in n-space and the rigidity of the hyperbolic space forms, Publ. Math. IHES 34 (1968) 53.

[50] P. Sarnak, The arithmetic and geometry of some hyperbolic three manifolds, Acta Math. 151 (1983) 253. 